\title{
分子马达集体运行机制及肌小节动态力学模型
}

\author{
殷跃红“，郭朝 \\ 上海交通大学机器人研究所, 机械系统与振动国家重点实验室, 上海 200240 \\ *E-mail: yhyin@sjtu.edu.cn
}

收稿日期: 2010-12-26; 接受日期: 2011-03-24

国家自然科学基金(批准号: 61075101/60643002)、国家重点基础研究发展计划(批准号: 2011CB013203)、国家高技术研究发展计划(批准 号: 2006AA04Z240)、上海市教委曙光计划(批准号: 07SG14)和上海交通大学医工交叉研究基金重大项目(批准号: YG2010ZD ${ }_{1} 01$ )资助

\begin{abstract}
摘要分子马达的集体协作运行机制尚未有效揭示清楚, 本文以骨骼肌中的肌小节为 研究对象, 利用非平衡态统计力学方法分析肌球蛋白分子马达的集体运行特性, 构建肌 小节系统的动力学方程和概率密度函数的 Fokker-Planck 方程, 推导出肌小节主动收缩力 学模型. 根据分子马达的实际状态对模型进行化简, 利用状态跃迁速率计算分子马达位移 变量的概率密度分布, 重点讨论了 $[A T P]$ 和负载力对概率密度、主动收缩力以及收缩速度 的影响, 分析可知随着 $[A T P]$ 的增大, 收缩力和收缩速度也逐渐增大, 在 $[A T P]$ 饱和后, 肌 小节的收缩力和收缩速度保持不变; 收缩速度随着负载力的增大逐渐减小到零; 收缩力 与肌小节的长度关系和粗细肌丝的有效作用长度有关, 随着有效长度减小而减小. 以上分 析与 Hill 描述的肌肉宏观特性比较吻合, 验证了模型的有效性.
\end{abstract}

关键词

分子马达

肌动蛋白

肌小节

集体特性

肌肉力学模型
分子马达是一类将化学能转化为机械能的纳米 分子机器, 随着生物纳米(Bio-NEMS) 技术的快速发 展, 分子马达的基础理论、人工分子马达的研制成为 当前的研究热点 ${ }^{[1 \sim 6]}$. 在国内, 分子马达研究取得重 要进展, 具有代表性的如: Ren 等人 ${ }^{[4]}$ 将纳米金属镍 丝与 $A T P$ 酶马达中的 $F_{1}$ 分子马达连接构成纳米推进 器, 通过设计调控磁场实现了对旋转分子马达的调 控. Gao 等人 ${ }^{[5]}$ 设计实现了金表面上具有固定偏心轴 的单分子转子及其大面积的有序组装. Qi 等人 ${ }^{[6]}$ 利用 分子组装技术将 $A T P$ 酶组装到磷脂修饰的聚合物微 胶囊上, 实现了 $A T P$ 的生物合成. 分子马达及其仿生 研究是目前纳米科技的核心领域之一, 它们之间的 协作机制是其中的难点问题 ${ }^{[7]}$.

肌球蛋白马达(Myosin II) 作为一种非持续性的
分子马达, 具有较小的占空比, 它是构成骨骼肌的主 要成分, 大量的肌球蛋白马达在细肌丝上协作运动 使得骨骼肌产生收缩, 开展肌球蛋白分子马达集体 特性研究是分析骨骼肌收缩原理的有效手段. 早在 1954 年, Huxley 等人 ${ }^{[8]}$ 便提出微观横桥模型来解释肌 肉收缩过程, 此后, 肌肉收缩机理研究得以迅速发展. 从物理学建模角度, Julicher 等人 ${ }^{[9]}$ 首次提出二态棘轮 模型分析分子马达的集体特性. Shu 等人 ${ }^{[10]}$ 则基于二 态棘轮模型讨论马达协作运动对 $A T P$ 水解动力学的 影响, 揭示 $A T P$ 浓度与自发振动之间关系. 从化学动 力学角度, Duke ${ }^{[11]}$ 提出了一个针对分子马达工作过 程的随机模型, 能在分子水平上解释肌肉稳态收缩 过程中的机械与热力学特性, 但是对分子马达系统 的空间结构缺少考虑, 不能体现肌肉整体收缩力学

英文版发表信息: Yin Y H, Guo Z. Collective mechanism of molecular motors and a dynamic mechanical model for sarcomere. Sci China Tech Sci, 2011, 54: 2130-2137, doi: 10.1007/s11431-011-4458-1 
特征. Lan 等人 ${ }^{[12]}$ 基于单个马达的构象变化情况和化 学反应速率构建了稳态时的肌肉收缩模型, 并以细 肌丝为对象研究了负载力与收缩速度关系. Lecarpentier 等人 ${ }^{[13]}$ 将分子马达的循环过程分成 6 个不同 状态, 基于 Huxley 模型分析骨骼肌力学特性. 物理 学研究主要对分子马达集体运行过程进行定性分析, 不能反映系统的实际力学特性; 从化学角度结合分 子马达的做功过程来分析肌肉收缩机理, 缺乏对肌 肉收缩过程的统一数学描述, 针对集体特性的定量 计算较少, 限制了模型的实际应用. 由于分子马达之 间相互影响，其集体运行特性不是各个马达作用的 简单叠加, 分子马达集体协作机制的研究还有待深 入, 因此, 在考虑肌球蛋白马达和肌动蛋白丝的实际 结构基础上, 从分子马达集体运行角度开展骨骼肌 收缩机理研究具有非常重要的意义.

本文以肌小节为研究对象开展肌球蛋白马达集 体协作特性分析, 主要结合肌小节的空间结构特征, 利用非平衡统计力学方法建立肌小节系统的动态力 学模型, 将定性分析与定量计算相结合, 讨论 ATP 浓 度对主动收缩力、收缩速度的影响, 分析主动收缩力 与肌肉长度、负载力与收缩速度之间的关系, 并结合 肌肉力检测实验来验证该数学模型的正确性.

\section{1 肌小节的体系结构}

如图 1 所示, 肌小节是肌肉收缩的最基本单位, 它沿轴向串联组成肌原纤维, 两端为 $\mathrm{Z}$ 线, 中间为 $\mathrm{M}$ 线. 肌小节主要包含粗肌丝和细肌丝: 其中粗肌丝 (Thick Filament) 由肌球蛋白(分子马达)叠加构成, 肌 球蛋白头部沿 6 个方向分布在粗肌丝表面; 细肌丝 (Thin Filament)是二聚体, 由两根肌动蛋白丝、原肌 球蛋白和肌钙蛋白组成. 当 $\mathrm{Ca}^{2+}$ 和肌钙蛋白结合后, 肌球蛋白和肌动蛋白结合并水解 $A T P$, 获取能量向 $\mathrm{M}$ 线方向弯曲. 大量的分子马达在细肌丝轨道上做 功, 使 $\mathrm{Z}$ 线向 $\mathrm{M}$ 线靠近, 肌小节产生收缩. 从肌小节 的横截面来看, 每根细肌丝周围有 3 根粗肌丝, 而每 根粗肌丝周围均布有 6 根细肌丝, 粗细肌丝的中心距 离约为 $23 \mathrm{~nm}^{[14]}$.

\section{2 肌小节系统建模分析}

\section{1 状态变量定义}

1 根粗肌丝和 6 根细肌丝构成的单元可以描述肌 小节的收缩特征, 由于肌小节的对称结构, 只需针对 半个肌小节进行建模分析, 如图 2 所示, 令粗肌丝的 长度为 $l_{\mathrm{m}}$, 一根粗肌丝上总的马达数目为 $n_{0}$, 细肌丝

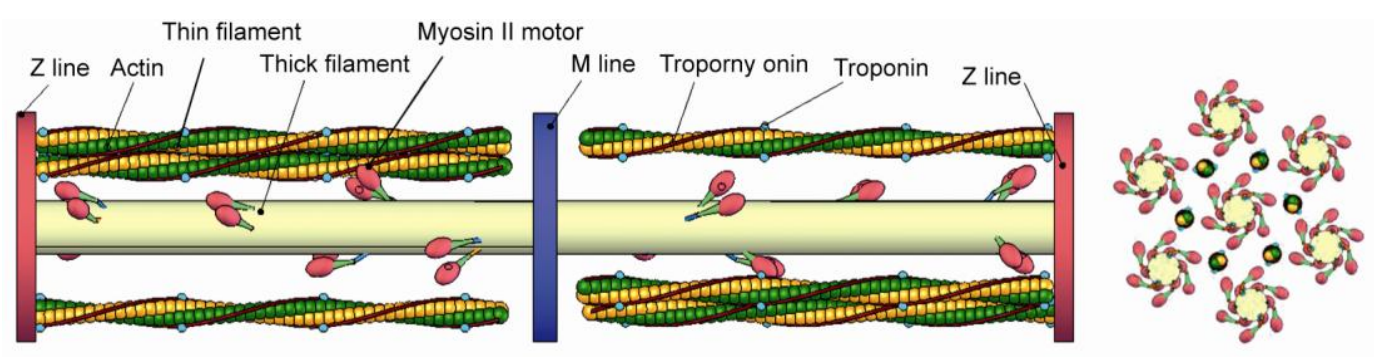

图 1 肌小节构成和肌小节的横截面

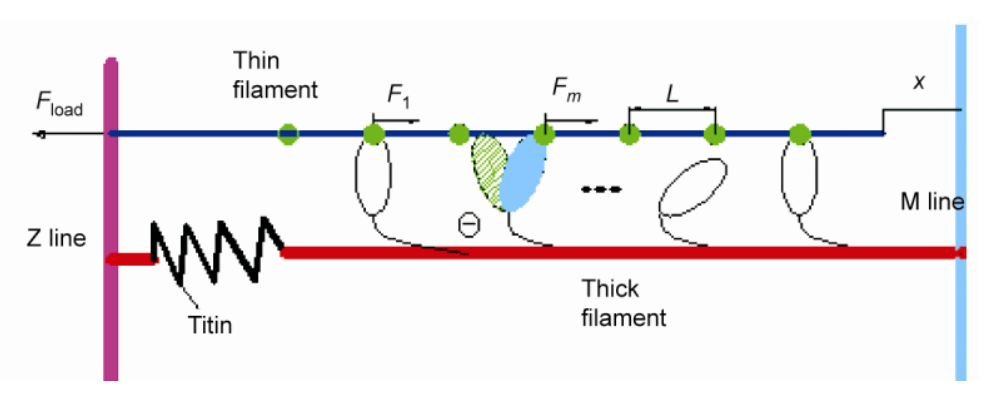

图 2 肌小节简化模型 
长度为 $l_{\mathrm{a}}$, 细肌丝上两个结合位点之间距离为 $L$.

对于粗肌丝上的任意一个肌球蛋白马达, 它的 工作周期可以分成结合并水解 $A T P$ 、吸附到细肌丝轨 道、定向运动和脱离轨道等几个过程, 每个过程对应 分子马达的一个化学状态, 分子马达在各个状态之 间跳转, 假设一个循环周期包含 $N$ 个状态, 其中第 $i$ 个状态用 $\sigma_{i}$ 表示, 经过一个周期分子马达水解一个 $A T P$ 并从一个结合位点跳到下一个结合位点, 前进 位移为 $L$. 在与肌动蛋白结合的过程中, 马达通过构 象变化推动细肌丝向 $\mathrm{M}$ 线运动, 令马达中心和细肌 丝相对于粗肌丝的前进位移 $x$ 为状态变量, $\rho_{i}(x, t)$ 表 示其中第 $m$ 个马达在 $t$ 时刻处于状态 $\sigma_{i}$ 时位移变量 $x$ 的概率密度.

\section{2 系统动力学方程}

分子马达在对细肌丝做功过程中, 主要受细肌 丝对它的负载力 $F_{\mathrm{e}}$ 、布朗力、主动力和阻尼力作用, 在过阻尼状态下, 动力学过程用 Langevin 方程表示:

$$
\gamma_{\mathrm{m}} \dot{x}(t)=\frac{\partial V_{i}(x, t)}{\partial x}-F_{\mathrm{e}}+\sqrt{2 \gamma_{\mathrm{m}} k_{\mathrm{B}} T} \cdot \varepsilon(t), \quad 1 \leqslant i \leqslant N,
$$

其中, $\gamma_{\mathrm{m}}$ 为分子马达的阻尼系数, $\gamma_{\mathrm{m}}=6 \pi \eta r_{\mathrm{m}}, \eta$ 为 水的黏性系数, $r_{\mathrm{m}}$ 为分子马达半径; 根据 Einstein-Stokes 关系, 分子马达在水溶液中的扩散系 数 $D=k_{\mathrm{B}} T / \gamma_{\mathrm{m}}, k_{\mathrm{B}}$ 为 Boltzmann 常数, $T$ 为热力学温 度; $\varepsilon(t)$ 为高斯白噪声, 满足 $\langle\varepsilon(t)\rangle=0,\left\langle\varepsilon(t), \varepsilon\left(t^{\prime}\right)\right\rangle=$ $\delta\left(t-t^{\prime}\right) . \quad V_{i}(x, t)$ 表示分子马达在 $\sigma_{i}$ 状态下沿 $x$ 方向 的主动作用势能, 满足 $V_{i}(x, t)=V_{i}(x+L, t)$.

细肌丝在向 $\mathrm{M}$ 线运动过程中, 同时受多个与之 结合的分子马达作用, 在 $\mathrm{Z}$ 线端面上还有负载力 $F_{\text {load }}$, 其 Langevin 方程为

$$
\gamma_{\mathrm{a}} \dot{x}(t)=\sum_{m=1}^{n_{0}} \frac{\partial V_{i_{m}}(x, t)}{\partial x}-F_{\text {load }}+\sqrt{2 \gamma_{\mathrm{a}} k_{\mathrm{B}} T} \cdot \varepsilon(t),
$$

其中, $\gamma_{\mathrm{a}}$ 为细肌丝的阻尼系数, $V_{i_{m}}(x, t)$ 表示第 $m$ 个 分子马达与细肌丝之间的作用势能.

\section{3 概率密度的 Fokker-Planck 方程}

由非平衡态统计力学原理, (1)式可转化为概率 密度函数 $\rho_{i}(x, t)$ 随时间演化的 Fokker-Planck 方程 ${ }^{[15]}$ :

$$
\frac{\partial \rho_{i}}{\partial t}=\underbrace{\frac{D}{k_{B} T} \frac{\partial}{\partial x}\left[\left(\frac{\partial V_{i}}{\partial x}-F_{e}\right) \rho_{i}\right]}_{\text {Active motion }}+\underbrace{D \frac{\partial^{2} \rho_{i}}{\partial x^{2}}}_{\text {Brownian motion }}+\underbrace{\sum_{j=1}^{N} k_{i j} \rho_{j}}_{\text {Chemical reations }} .
$$

方程(3)表示分子马达在主动力、负载力及布朗 力作用下的随机演化过程, 该方程同时也反映了分 子马达化学反应动力学特性. 其中, $k_{i j}$ 表示分子马 达从状态 $i$ 跳转到状态 $j$ 时的跃迁速率.

如图 3 所示, 在两个化学状态 $\left[\sigma_{i}, \sigma_{i+1}\right]$ 之间, 令 蛋白马达从 $\sigma_{i}$ 转移到状态 $\sigma_{i+1}$ 时跃迁速率为 $k_{i}$, 从 状态 $\sigma_{i+1}$ 回到状态 $\sigma_{i}$ 的跃迁速率为 $k_{-i}$. 一个循环周 期分子马达水解一个 $A T P$, 获得的自由能为 $\Delta G=$ $20 k_{\mathrm{B}} T$, 当系统达到稳定状态后, 跃迁速率满足细致 平衡条件:

$$
\begin{gathered}
\frac{k_{i}}{k_{-i}}=\exp \left(V_{i}-V_{i+1} / k_{\mathrm{B}} T\right), \quad 1 \leqslant i \leqslant N-1, \\
\frac{k_{N \rightarrow 1}}{k_{1 \rightarrow N}}=\exp \left\{\left[V_{N}-\left(V_{1}+\Delta G\right)\right] / k_{\mathrm{B}} T\right\} .
\end{gathered}
$$

由 $V_{i}(x)$ 的性质, $k_{i}$ 也满足周期性条件: $k_{i}(x+L)=k_{i}(x)$.

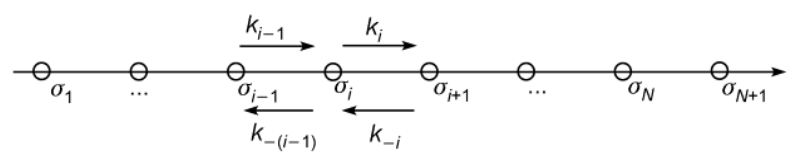

图 3 两个化学状态间的跃迁速率

定义分子马达处在 $i$ 状态时, 沿着 $x$ 方向的概率 流:

$$
J_{i}(x)=-D\left(\frac{\left(V_{i}^{\prime}-F_{\mathrm{e}}\right)}{k_{\mathrm{B}} T} \rho_{i}+\frac{\partial \rho_{i}}{\partial x}\right) .
$$

方程(3)化简为

$$
\frac{\partial \rho_{i}}{\partial t}=-\frac{\partial J_{i}}{\partial x}+\sum_{j=1}^{N} k_{i j} \rho_{j} .
$$

为了计算概率密度分布函数 $\rho_{i}(x, t)$, 对(7)式进 行离散化, 如图 4 所示, 将分子马达在 $x$ 方向的位移 区间 $[0, L]$ 划分为 $M$ 等份, $\Delta x=x_{n}-x_{n-1}=L / M$, 马 达从 $x_{n}$ 移动到下一个位置 $x_{n+1}$ 时跃迁速率为 $F_{n}$, 从位 置 $x_{n+1}$ 回到位置 $x_{n}$ 的跃迁速率为 $B_{n}$. 在 $\left[x_{n}, x_{n+1}\right]$ 内的 概率为

$$
P_{i}^{n+1}(x, t)=\int_{x_{n}}^{x_{n+1}} \rho_{i}(x, t) \mathrm{d} x=\rho_{i}^{n+1}(x, t) \Delta x .
$$




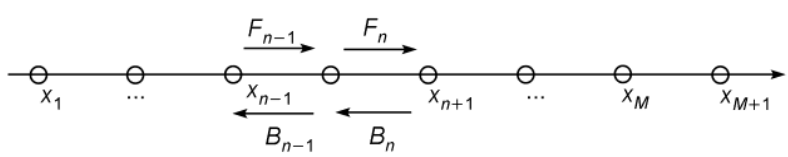

图 4 两个位置间的跃迁速率

方程(7)转化为概率密度的主方程:

$$
\begin{aligned}
\frac{\mathrm{d} P_{i}^{n}}{\mathrm{~d} t}=J_{i}^{n-1} & -J_{i}^{n}+\sum_{j \neq i}^{N}\left(k_{j i} P_{j}^{n}-k_{i j} P_{i}^{n}\right), 1 \leqslant n \leqslant M ; 1 \leqslant i \leqslant N, \\
\frac{\mathrm{d} P_{i}^{n}}{\mathrm{~d} t} & =\left(F_{i}^{n-1} P_{i}^{n-1}-B_{i}^{n} P_{i}^{n}\right)-\left(F_{i}^{n} P_{i}^{n}-B_{i}^{n+1} P_{i}^{n+1}\right) \\
& +\sum_{j \neq i}^{N}\left(k_{j i} P_{j}^{n}-k_{i j} P_{i}^{n}\right) .
\end{aligned}
$$

分子马达在两个位置 $\left[x_{n}, x_{n+1}\right.$ 之间的跃迁速率满 足 $^{[16]}$ :

$$
\left\{\begin{array}{l}
B_{i}^{n+1}=\frac{D}{\Delta x^{2}}\left(\frac{\Delta \phi_{i}^{n} / k_{\mathrm{B}} T}{\exp \left(\Delta \phi_{i}^{n} / k_{\mathrm{B}} T\right)-1}\right), \\
F_{i}^{n}=\frac{D}{\Delta x^{2}}\left(\frac{-\Delta \phi_{i}^{n} / k_{\mathrm{B}} T}{\exp \left(-\Delta \phi_{i}^{n} / k_{\mathrm{B}} T\right)-1}\right),
\end{array}\right.
$$

其中, $\phi_{i}(x)=V_{i}(x)-x \cdot F_{\mathrm{e}}$.

\section{4 主动收缩力与收缩速度}

若 $\mathrm{Ca}^{2+}$ 浓度足够大时, 则细肌丝上的结合位点 均被打开, 此时肌球蛋白可与肌动蛋白充分结合. 由 方程(3)可以确定分子马达在任一时刻的状态, 由于 化学反应和构象变化过程同步进行, 每个分子马达 的状态和它头部的弯曲程度相对应, 马达头部不同 的弯曲程度对应着肌球蛋白马达对细肌丝不同的作 用力, 因此肌小节上所有分子马达的主动收缩力为

$$
F=\frac{A \alpha n_{0} c}{s} \int_{0}^{L} x \rho(x, t) \mathrm{d} x,
$$

其中, $c$ 为分子马达的弹性系数, $\alpha$ 表示粗肌丝与细肌 丝的交叠(Overlap)程度, 当他们处于最佳的交叠位 置时 $\alpha=1$ 所有分子马达均可以和肌动蛋白结合而做 功. $A$ 为肌小节的横截面积, $s$ 为粗肌丝周围 6 根细肌

$$
\dot{P}_{i}^{\prime}=\left[\begin{array}{ccc}
-\left(k_{-5}+k_{1}\right) & k_{-1} & \\
k_{1} & -\left(k_{-1}+k_{2}\right) & k_{-2} \\
& k_{2} & -\left(k_{-2}+k_{3}\right) \\
& & k_{3} \\
k_{-5} & &
\end{array}\right.
$$

丝构成的六边形面积, $A / s$ 表示肌小节上所包含的粗 肌丝数目.

概率密度满足的约束条件:

$$
\rho(0, t)=\rho(L, t)=0, \quad \int_{0}^{L} \rho(x, t) \mathrm{d} x=1 .
$$

方程组

$$
\left\{\begin{array}{l}
\frac{\partial \rho_{i}}{\partial t}=\frac{D}{k_{\mathrm{B}} T} \frac{\partial}{\partial x}\left[\left(\frac{\partial V_{i}}{\partial x}-F_{\mathrm{e}}\right) \rho_{i}\right]+D \frac{\partial^{2} \rho_{i}}{\partial x^{2}}+\sum_{j=1}^{N} k_{i j} \rho_{j}, \\
F=\frac{A \alpha n_{0} c}{s} \int_{0}^{L} x \rho(x, t) \mathrm{d} x .
\end{array}\right.
$$

构成了肌小节系统的动态力学模型, 当确定各个参 数后, 可以计算肌小节的主动收缩力, 将在后面展开 讨论.

稳态情况下, 分子马达处在状态 $\sigma_{i}$ 时的运动速 度：

$$
v_{i}=L J_{i}(x) .
$$

肌小节的收缩速度, 即细肌丝相对于粗肌丝的 平均运动速度:

$$
\nu=\frac{1}{N} \sum_{i=1}^{N} v_{i}=\frac{1}{N} \sum_{i=1}^{N} L J_{i} .
$$

\section{3 模型简化分析}

由于肌球蛋白马达运动过程中力与化学的耦合 关系, 马达前进位移的改变伴随着化学状态的变化. 首先分析化学状态的变化情况, 将马达的一个循环 周期分为 5 个状态, 其中分子马达做功过程包含 $A \cdot M \cdot A D P \cdot P, A \cdot M \cdot A D P, A \cdot M$ 三个状态, 其所 处概率分别为 $p_{1}^{\prime}, p_{2}^{\prime}, p_{3}^{\prime}$; 分子马达水解 $A T P$ 过程包 含 $M \cdot A T P, M \cdot A D P \cdot P_{i}$ 两个状态, 所处概率分别为 $p_{4}^{\prime}, p_{5}^{\prime}$. 如图 5 所示.

根据(5)式, 各状态之间的概率转移方程:

$$
\dot{P}_{i}^{\prime}=k_{i-1} P_{i-1}^{\prime}-k_{-(i-1)} P_{i}^{\prime}-k_{i} P_{i}^{\prime}+k_{-i} P_{i+1}^{\prime} .
$$

由 $N=5$ 可进一步化简如下:

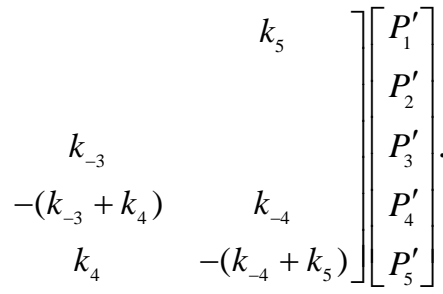


根据约束条件: $P_{1}^{\prime}+P_{2}^{\prime}+P_{3}^{\prime}+P_{4}^{\prime}+P_{5}^{\prime}=1$.

由文献[17]可知, 在马达做功过程中, 其状态跃 迁速率 $k_{i}$ 受负载力的影响, 而在马达水解 $A T P$ 阶段却 不受负载力的影响, 其速率常数只与自由能相关; 根 据 Arrhenius 公式, 定义 $k_{i}=k_{i}^{0} \exp \left[\left(-d_{i} \cdot F_{\mathrm{e}}\right) / k_{\mathrm{B}} T\right]$, $i=1,2 ; k_{i}=k_{i}^{0}, i=3,4,5 ; d_{i}$ 为马达头部构象变化 前进的位移, $k_{i}^{0}$ 为零负载情况下的初始跃迁速率, 由分子马达自由能变化值决定, 利用细致平衡条件 (4)式可计算 $k_{-i}$. 马达各个状态的初始 $k_{i}^{0[12]}$ 如图 6 所 示.

肌球蛋白只有和肌动蛋白结合后才开始做功, 并对细肌丝产生作用力, 进一步将分子马达的状态 数简化为 2 个, 状态 1 表示马达结合状态, 状态 2 为 马达分离状态. 如下所示:

$$
\begin{aligned}
& P_{1} \quad P_{2} \\
& 1 \underset{k_{12}^{-}}{\stackrel{k_{12}^{+}}{\rightleftarrows}} 2 \underset{k_{21}^{-}}{\stackrel{k_{21}^{+}}{\rightleftarrows}} 1
\end{aligned}
$$

两状态之间转移的跃迁速率 $k_{12}, k_{21}$ 满足 ${ }^{[15]}: k_{12}=$ $k_{12}^{+}+k_{21}^{-}, \quad k_{21}=k_{21}^{+}+k_{12}^{-}$, 其 中, $\frac{1}{k_{12}^{+}} \approx \frac{1}{k_{1}}+\frac{1}{k_{2}}+\frac{1}{k_{5}}$, $\frac{1}{k_{12}^{-}} \approx \frac{1}{k_{-1}}+\frac{1}{k_{-2}}+\frac{1}{k_{-5}}, \frac{1}{k_{21}^{+}} \approx \frac{1}{k_{4}}+\frac{1}{k_{3}[A T P]}, \frac{1}{k_{21}^{-}} \approx \frac{1}{k_{-3}}+$ ${\frac{1}{k_{-4}}}^{[18]},[A T P]$ 表示参与反应的 $A T P$ 浓度.

由上述分析可知, 结合 5 个状态情况下的化学跃 迁速率实验值, 便可计算肌球蛋白马达在两种状态 下的化学跃迁速率, 并可进一步求得概率密度 $\rho_{i}(x, t)$ 沿着位移 $x$ 方向的分布.

根据马达主动势能满足非对称性周期势能特征, 将沿 $x$ 方向的势能函数定义为 ${ }^{[19,20]}$ :

$$
\left\{\begin{array}{l}
V_{1}(x)=\Delta G\left[\sin \left(\frac{2 \pi x}{L}\right)+\frac{1}{4} \sin \left(\frac{4 \pi x}{L}\right)\right], \\
V_{2}(x)=\text { constant. }
\end{array}\right.
$$

分子马达在两个状态之间转移, 方程(3)化简为

$$
\left\{\begin{aligned}
\frac{\partial \rho_{1}}{\partial t}= & \frac{D}{k_{\mathrm{B}} T} \frac{\partial}{\partial x}\left[\left(\frac{\partial V_{1}}{\partial x}-F_{\mathrm{e}}\right) \rho_{1}\right] \\
& +D \frac{\partial^{2} \rho_{1}}{\partial x^{2}}-k_{12} \rho_{1}+k_{21} \rho_{2}, \\
\frac{\partial \rho_{2}}{\partial t}= & \frac{D}{k_{\mathrm{B}} T} \frac{\partial}{\partial x}\left[\left(\frac{\partial V_{2}}{\partial x}\right) \rho_{2}\right] \\
& +D \frac{\partial^{2} \rho_{2}}{\partial x^{2}}+k_{12} \rho_{1}-k_{21} \rho_{2} .
\end{aligned}\right.
$$

根据(8)式, (18)式化简为主方程:

$$
\left\{\begin{aligned}
\frac{\mathrm{d} P_{1}^{n}}{\mathrm{~d} t}= & \left(F_{1}^{n-1} P_{1}^{n-1}-B_{1}^{n} P_{1}^{n}\right)-\left(F_{1}^{n} P_{1}^{n}-B_{1}^{n+1} P_{1}^{n+1}\right) \\
& +\left(k_{21} P_{2}^{n}-k_{12} P_{1}^{n}\right), \\
\frac{\mathrm{d} P_{2}^{n}}{\mathrm{~d} t}= & \left(F_{2}^{n-1} P_{2}^{n-1}-B_{2}^{n} P_{2}^{n}\right)-\left(F_{2}^{n} P_{2}^{n}-B_{2}^{n+1} P_{2}^{n+1}\right) \\
& +\left(k_{21} P_{1}^{n}-k_{12} P_{2}^{n}\right) .
\end{aligned}\right.
$$

$L_{i}=$

$\left[\begin{array}{cccccc}-\left(B_{i}^{1}+F_{i}^{1}\right) & B_{i}^{2} & & & & F_{i}^{M} \\ F_{i}^{1} & -\left(B_{i}^{2}+F_{i}^{2}\right) & B_{i}^{3} & & & \\ & F_{i}^{2} & -\left(B_{i}^{3}+F_{i}^{3}\right) & B_{i}^{4} & & \vdots \\ & & F_{i}^{3} & \ddots & \ddots & \\ & & & \ddots & \ddots & B_{i}^{M} \\ B_{i}^{1} & & & & F_{i}^{M-1} & -\left(B_{i}^{M}+F_{i}^{M}\right)\end{array}\right]$, $i=1,2$,

(19)式可化简为

$$
\frac{\mathrm{d}}{\mathrm{d} t}\left[\begin{array}{l}
P_{1}^{n} \\
P_{2}^{n}
\end{array}\right]=\left[\begin{array}{cc}
L_{1}-k_{12} & k_{21} \\
k_{12} & L_{2}-k_{21}
\end{array}\right]\left[\begin{array}{l}
P_{1}^{n} \\
P_{2}^{n}
\end{array}\right] .
$$

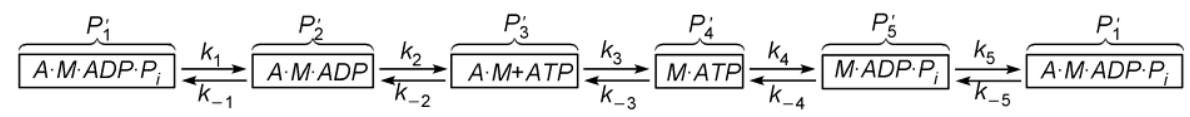

图 5 一个循环周期的状态划分

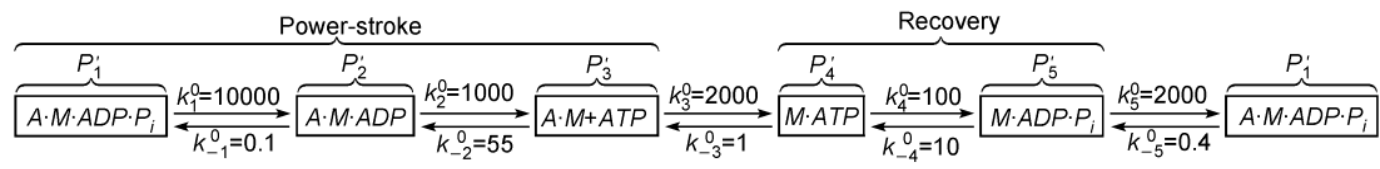

图 6 状态跃迁速率 $\boldsymbol{k}_{i}$ 的初始值 


\section{4 数值计算与结果讨论}

\section{1 概率密度分布及其影响因素}

所用参数如表 1 所示, 针对方程组(20), 取 $M=80$, 计算分子马达沿着 $x$ 轴方向的概率分布函数. Finer 等 人 $^{[21]}$ 通过光铌实验测定分子马达头部在释放 $P_{i}$ 和 $A D P$ 过程中, 推动细肌丝前进的位移量 $d_{i}$ 分别为 4 和 $6 \mathrm{~nm}$. 结合过程中的概率 $P_{1}=k_{12} /\left(k_{12}+k_{21}\right)$, 由于 状态跃迁速率 $k_{i j}$ 主要受 $[A T P]$ 和负载力 $F_{\mathrm{e}}$ 影响, 概率 $P_{1}$ 也会随着 $[A T P]$ 和负载力的改变而改变. 计算同一 负载、不同 $[A T P]$ 下概率密度的变化情况如图 7 所示, 其中 $F_{\mathrm{e}}=1 \mathrm{pN},[A T P]$ 分别为 $0.1,0.5,1,10 \mu \mathrm{M}$, 由图可 看出概率密度满足 Boltzmann 分布规律, 并且随着 $[A T P]$ 增大, 概率 $P_{1}$ 也逐渐增大, 当 $[A T P]$ 小于 $1 \mu \mathrm{M}$ 时, 结合概率增大较快; 而当 $[A T P]$ 大于 $1 \mu \mathrm{M}$ 后, $P_{1}$ 增幅减小, 直到 $[A T P]$ 饱和后, 马达与细肌丝结合的 概率将不再改变.

\section{表 1 特征参数 ${ }^{[12 ~ 14]}$}

\begin{tabular}{ccccc}
\hline$l_{\mathrm{s}}$ & $l_{\mathrm{m}}$ & $l_{\mathrm{a}}$ & $s$ & $n_{0}$ \\
\hline $2500 \mathrm{~nm}$ & $1650 \mathrm{~nm}$ & $1300 \mathrm{~nm}$ & $1.374 \times 10^{3} \mathrm{~nm}^{2}$ & 150 \\
\hline \hline$c$ & $D$ & $k_{\mathrm{B}} T$ & $L$ & $r_{m}$ \\
\hline $0.7 \mathrm{pN} / \mathrm{nm}$ & $5.47 \times 10^{7} \mathrm{~nm}^{2} / \mathrm{s}$ & $4.1 \mathrm{pN} \mathrm{nm}$ & $36 \mathrm{~nm}$ & $4 \mathrm{~nm}$ \\
\hline
\end{tabular}

图 8 所示为相同的 $[A T P]$ 、不同负载情况下的概 率密度分布情况. 取 $M=80,[A T P]=1 \mu \mathrm{M}$, 负载力 $F_{\mathrm{e}}=1,2,3,4 \mathrm{pN}$, 结合概率 $P_{1}$ 随着负载力的增加而逐 渐增大. 说明在负载力增加的情况下, 分子马达延长 了与细肌丝结合的时间, 结合概率增大, 分子马达头 部能够产生更大的应力以抵消负载力.

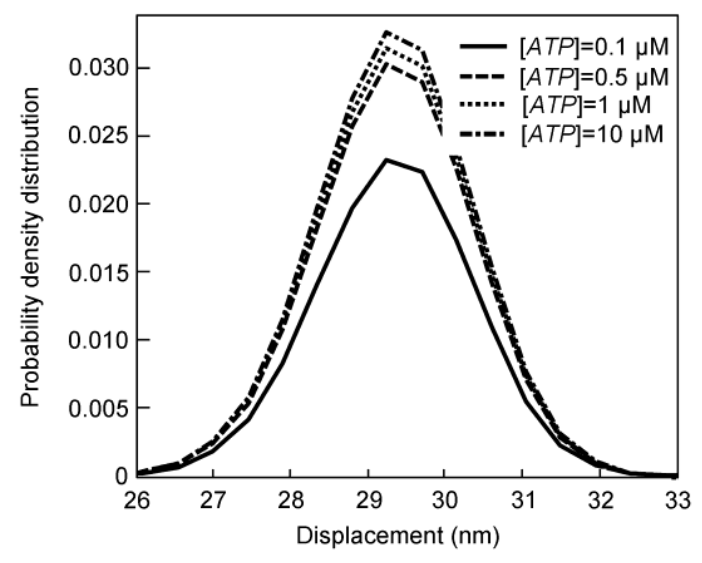

图 7 相同负载、不同 $[A T P]$ 时的概率分布函数

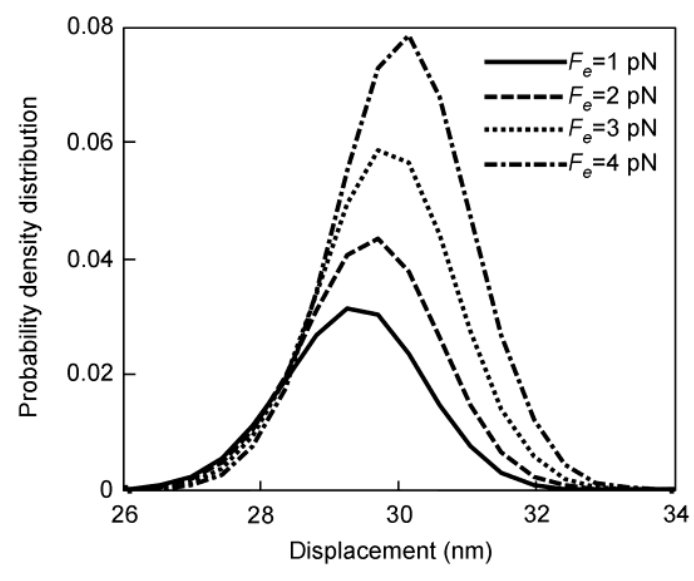

图 8 不同负载、相同 $[A T P]$ 时的概率分布函数

\section{2 主动收缩力及影响因素}

根据(10)式可知, 肌小节上的主动收缩力主要取 决于分子马达数目 $n_{0}$ 、弹性系数 $c$ 、粗细肌丝的交叠 程度 $\alpha$ 、横截面积 $A$ 以及概率密度分布函数. 首先, 当 外部条件负载力 $F_{\mathrm{e}}$ 和 $[A T P]$ 的改变时, 结合状态下的 概率密度函数分布随之改变, 从而影响主动收缩力. 图 9 为一根粗肌丝产生的主动收缩力与 $[A T P]$ 之间关 系, 当粗细肌丝的交叠程度处于最佳位置时 $\alpha=1$, 取 肌小节的端面负载力为 $30 \mathrm{pN}$, 从图 9 中可知, 随着 $[A T P]$ 增大, 主动收缩力逐渐增大, 开始时增速较快, 当 $[A T P]$ 饱和后, 收缩力将不再增加. 说明增加 $[A T P]$, 分子马达与 $A T P$ 的结合速率加快, 产生的主动收缩 力增大, 其最大值可达 $440 \mathrm{pN}$. 其次, 增大分子马达 数目 $n_{0}$ 以及弹性系数 $c$, 主动收缩力也会增大, Lecarpentier 等人 ${ }^{[13]}$ 实验测量了两种类型肌肉的最大 等容收缩力, 先测量 $1 \mathrm{~mm}^{2}$ 小腿比目鱼肌的最大主动 收缩力为 $110.2 \mathrm{mN}$, 据粗肌丝周围 6 根细肌丝构成的 6 边形面积 $s$, 可知 $1 \mathrm{~mm}^{2}$ 上的粗肌丝个数约为 $7.28 \times 10^{8}, 1$ 根粗肌丝产生的收缩力约等于 $151.4 \mathrm{pN}$; 然后测量小腿趾长伸肌上单位面积的收缩力为 85.1 $\mathrm{mN} / \mathrm{mm}^{2}$, 则 1 根粗肌丝的最大收缩力约等于 $116.9 \mathrm{pN}$. 可见, 两种类型的肌肉会产生不同的最大收缩力, 这 主要是由于分子马达数目 $n_{0}$ 及弹性系数 $c$ 不同所导致. 因此对于一定的 ATP 浓度和负载力, 由(10)式可计算 出肌肉的实验测定值. 此外, 对于肌小节来讲, 横截 面积越大, 则包含的粗肌丝数目越多, 产生的肌肉力 也越大, 肌肉力和截截面积成正比例关系. 交叠程度 $\alpha$ 主要受肌小节长度的影响, 将在下面讨论. 
由于肌小节结构的对称性，定义 $\alpha=\mathrm{e}^{-\left(l-l_{m}\right)^{2} / \sigma^{2}}$, $l_{\mathrm{m}}$ 为肌小节的最佳有效长度, $\sigma$ 表示收缩力大小的倾 斜程度, 取 $\sigma^{2}=0.5$, 最大主动收缩力 $F_{\mathrm{m}}$ 分别等于 120 , $140,160,180 \mathrm{pN}$, 肌小节长度与收缩力之间关系如 图 10 所示, 当 $l<l_{\mathrm{m}}$ 时, 粗肌丝与细肌丝之间产生重叠, 使部分肌球蛋白马达不能和肌动蛋白结合, 收缩力 $F$ 随着长度的减小逐渐减小; 而当肌小节长度超过它 的最佳长度 $l>l_{\mathrm{m}}$ 时, 也会导致部分肌球蛋白不能和肌 动蛋白结合, 收缩力 $F$ 开始逐渐减小. 计算结果和文

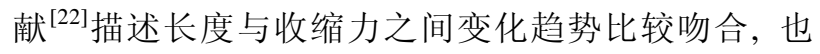
符合 Hill 描述的肌肉宏观力学特性 ${ }^{[23]}$.

\section{3 收缩速度及其影响因素}

根据(13)式可知, 稳态情况下肌小节的收缩速度 主要由跃迁速率决定, 因此, 收缩速度主要受 $[A T P]$ 和负载力的影响. 图 11 为不同 $[A T P]$ 情况下, 分子马

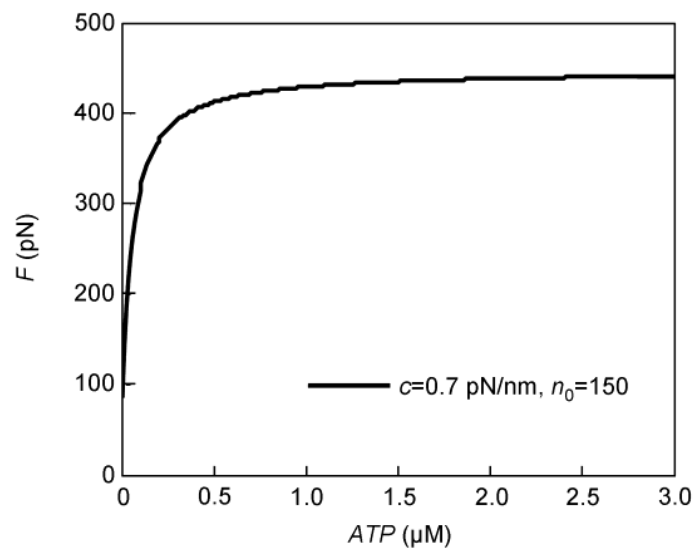

图 $9[A T P]$ 与主动收缩力之间关系

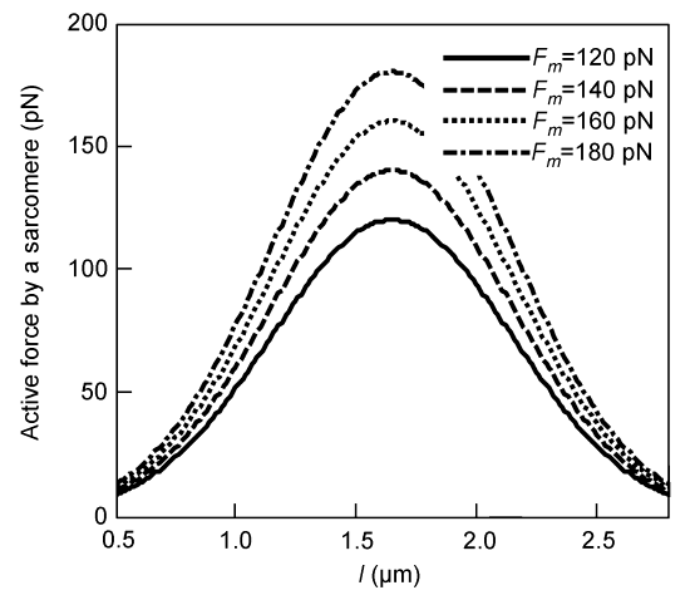

图 10 肌小节长度与主动收缩力之间关系
达上负载力 $F_{\mathrm{e}}$ 与收缩速度之间关系. 当 $[A T P]=0.1$ $\mu \mathrm{M}$ 时, 负载力从零逐渐增加, 收缩速度则逐渐减小 到零, 负载力在 2 6 $\mathrm{pN}$ 内时, 收缩速度与负载力之 间呈反比例关系，当负载增加到 $6 \mathrm{pN}$ 后，马达停止 向前运动, 较大的负载使马达无法推动细肌丝前进. 图中描述的收缩速度与负载力之间性质与 Hill 描述 的肌肉宏观力学属性一致 ${ }^{[23]}$.

图 12 为 $[A T P]$ 与收缩速度之间关系, 负载力 $F_{\mathrm{e}}=1$ $\mathrm{pN}$, 由图可知, 当增大 $[A T P]$ 时, 开始阶段收缩速度 增加较快, 在 $[A T P]$ 增加到 $10 \mu \mathrm{M}$ 后, 速度不再增加 并趋向稳定, 大小约 $2900 \mathrm{~nm} / \mathrm{s}$, 参与反应的 $[A T P]$ 与 反应速率之间满足 Michaelis-Menten 关系.

\section{5 结论}

本文通过分析肌球蛋白马达的集体运行特性研 究了骨骼肌微观力学原理, 并利用非平衡态统计力

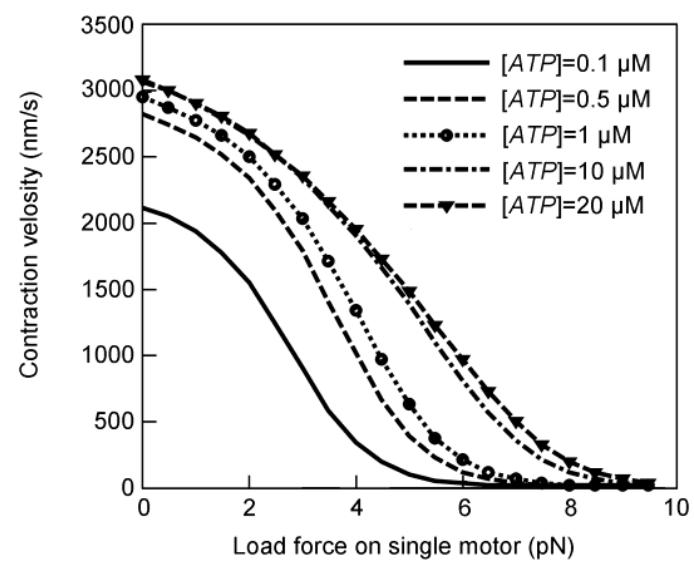

图 11 负载力与收缩速度之间关系

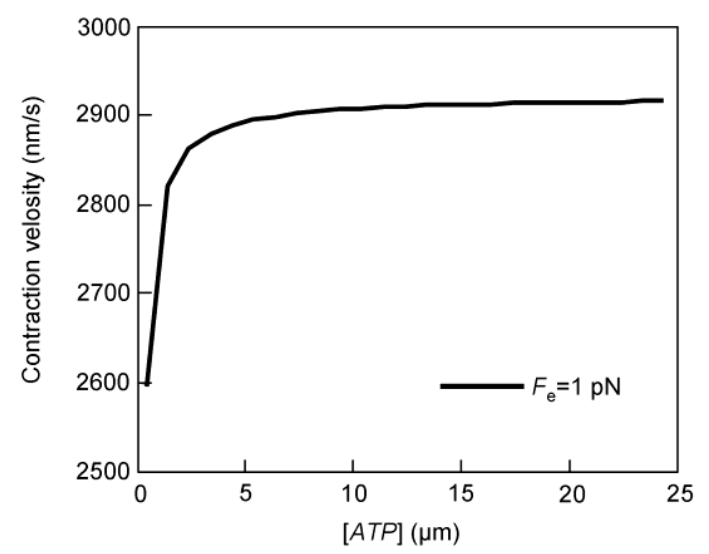

图 $12[A T P]$ 与收缩速度之间关系 
学方法建立了肌小节动态力学模型. 数值计算可知, 肌小节的主动收缩力和分子马达数目、弹性系数、横 截面积、以及外部的负载力和 $[A T P]$ 等因素相关; 在 一定的分子马达数目、横截面积及弹性系数情况下, 随着 $[A T P]$ 增加, 肌肉主动收缩力逐渐增大, 而随着 肌小节的长度增加, 主动收缩力先增大后减小; 肌小
节的收缩速度随着 $[A T P]$ 增加而逐渐增大, 而当 $[A T P]$ 饱和后收缩速度不再改变; 并随着负载力的增 加而减小, 直到为零. 以上分析一方面可揭示肌肉收 缩的微观机理，同时也为人肌肉宏观特性研究提供 理论指导，分子马达的集体特性同时受到钙离子浓 度的影响, 需要在以后深入展开.

\section{参考文献}

1 郭朝, 殷跃红, 肌球蛋白分子马达的多力场耦合机理分析. 科学通报, 2010, 55: 2675-2682

2 Van Delden R A, Ter Wiel M K J, Pollard M M, et al. Unidirectional molecular motor on a gold surface. Nature, 2005, 437: 1337-1340

3 Guo Z, Yin Y H. Casimir effect on adhesion interaction between myosin molecular motor and actin filament. Int J Nanosyst, 2010, 3: 9-15

4 Ren Q, Zhao Y P, Yue J C, et al. Biological application of multi-component nanowires in hybrid devices powered by F1-ATPase motors. Biomed Microdevices, 2006, 8: 201-208

5 Gao L, Liu Q, Zhang Y Y, et al. Constructing an array of anchored single-molecule rotors on gold surfaces. Phys Rev Lett, 2008, 101: 197209

6 Qi W, Duan L, Wang K W, et al. Motor protein CF0F1 reconstituted in lipid-coated hemoglobin microcapsules for ATP synthesis. Adv Mater, 2008, 20: 601-605

7 Vermeulen K C, Stienen G J M, Schmidt C F. Cooperative behavior of molecular motors. J Muscle Res Cell Motil, 2002, 23: 71-79

8 Huxley A F, Niedergerke R. Structural changes in muscle during contraction. Nature, 1954, 173: 971-973

9 Julicher F, Ajdari A, Prost J. Modeling molecular motors. Rev Mod Phys, 1997, 69: 1269-1281

10 Shu Y G, Shi H L. Cooperative effects on the kinetics of ATP hydrolysis in collective molecular motors. Phys Rev E, 2004, 69: 021912

11 Duke T A J. Molecular model of muscle contraction. Proc Natl Acad Sci USA, 1999, 96: 2770-2775

12 Lan G, Sun S X. Dynamics of myosin-driven skeletal muscle contraction: I. Steady-state force generation. Biophys J, 2005, 88: 4107-4117

13 Lecarpentier Y, Blanc F X, Quillard J, et al. Statistical mechanics of myosin molecular motors in skeletal muscles. J Theor Biol, 2005, 235: 381-392

14 Rayment I, Holden H M, Whittaker M, et al. Structure of the actin-myosin complex and its implications for muscle contraction. Science, 1993, 261: 56-65

15 Xing J, Wang H, Oster G. From continuum Fokker-Planck models to discrete kinetic models. Biophys J, 2005, 89: 1551-1563

16 Wang H, Peskin C S, Elston T C. A robust numerical algorithm for studying biomolecular transport processes. J Theor Biol, 2003, 221: 491-511

17 Veigel C, Molloy J E, Schmitz S, et al. Load-dependent kinetics of force production by smooth muscle myosin measured with optical tweezers. Nat cell biol, 2003, 5: 980-986

18 David K, Carlos B. The mechanochemistry of molecular motors. Biophys J, 2000, 78: 541-556

19 Shu Y G, Yue J C, Ou-Yang Z C. F0F1-ATPase, rotary motor and biosensor. Nanoscale, 2010, 2: 1284-1293

20 Reimann P. Brownian motors: Noisy transport far from equilibrium. Phys Rep, 2002, 361: 57-265

21 Finer J T, Simmons R M, Spudich J A. Single myosin molecule mechanics: Pico Newton forces and nano metre steps. Nature, 1994, 368: $113-119$

22 Shimamoto Y, Kono F, Suzuki M, et al. Nonlinear force-length relationship in the ADP-induced contraction of skeletal myofibrils. Biophys J, 2007, 93: 4330-4341

23 Hill A V. The heat of shortening and the dynamic constants of muscle. Proc R Soc Lond B, 1938, 126: 136-195 